\title{
CRUISING THE DINOSAUR FREEWAY
}

COTTON, William, D., National Radio Astronomy Observatory, 520 Edgemont Road, Charlottesville, VA 22903-2475 U.S.A. HUNT, Adrian P., Mesa Technical College, 911 South Tenth St., Tucumcari, NM 88401, U.S.A. COTTON, Jennifer E.,Charlottesville High School, 1400 Melbourne Road, Charlottesville, VA 22901, U.S.A.

Studying the trackways of extinct animals is one of the few ways to study social behaviors, especially herding, in these animals. Lockley, M. and A. P. Hunt. (1995. Dinosaur Tracks, Columbia University Press, New York) discuss a tracksite at Mosquero Creek, New Mexico which shows a large number of individuals of ichnogenus Caririchnium (a presumed iguanodontid) moving in apparently organized groups which they interpreted as herds. The tracks are all assigned to Caririchnium but different groups had different size footprints. In addition, the consistency of the track depths indicated that the tracks were made in a relatively short time span. We have further studied one of these groups analyzing the direction and speed of motion for consistency with the herding hypothesis. Speeds were determined using the method of Alexander, R. M. (1989. Dynamics of Dinosaurs \& Other Extinct Giants, Columbia University Press, New York). This site is part of an extended trackbearing horizion known as the "Dinosaur Freeway". While there are large uncertainties in the absolute speeds determined using this method, the relative speeds should be accurate.

We measured positions, sizes and direction of motion of footprints in 16 trackways which were unambiguous and consisted of three or more consecutive pes imprints. These trackways have a relatively narrow distribution of pes impression lengths indicating a rather narrow age distribution. Lockley and Hunt 1995 interpret these tracks as those of yearling iguanodontids. Most of the trackways measured had dimensionless speeds (as defined by Alexander, 1989) in the range of 0.3 to 0.5 which is significantly slower than the division between walking and running of 0.7 given by Alexander, 1986. The trackmakers were moving at a deliberate but not fast pace. The measured speeds and directions of motion (Figure 1) cluster around a well defined central value with significant dispersions in speed and direction. At this slow speed, some variation in speed and direction are expected and the observed distribution is consistent with herding behavior. In addition, there is a correlation (correlation coefficient $=-0.67$ ) between footprint size and dimensionless speed in the sense that the larger animals were exerting less effort than the smaller animals but were moving at approximately the same physical speed. We believe that these trackways represent a herd of yearling iguanodontids.

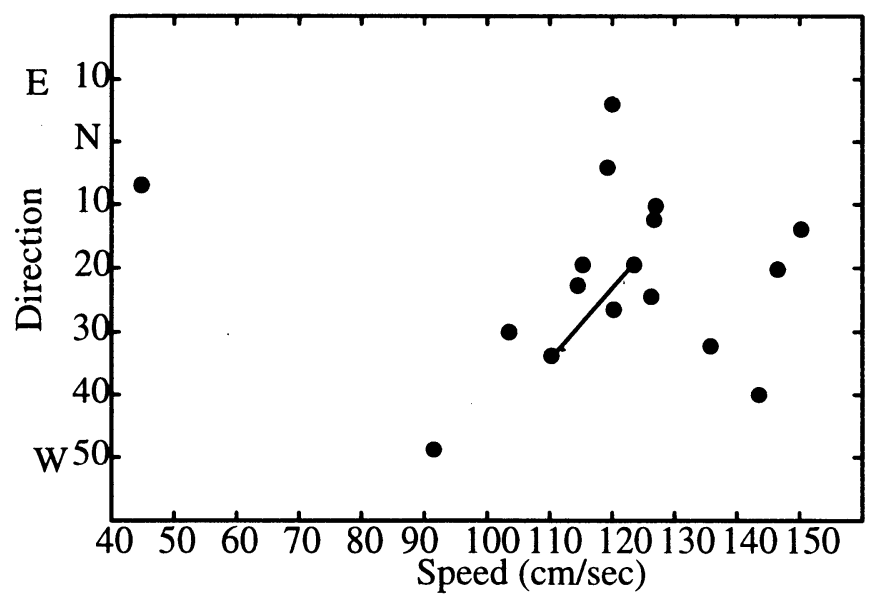

Figure 1: Speed and direction of motion of Caririchnium tracks at the Mosquero Creek tracksite. 